\title{
INGESTIBILITÉ COMPARÉE DE L'AVOINE DÉCORTIQUÉE, DU MAÏS ET DE L'ORGE DANS LES ALIMENTS CONGENTRÉS POUR AGNEAUX PRÉCOCEMENT SEVRÉS
}

\author{
(․ MOLENAT, M. THERIEZ et A. BBEN ABDELJELIL \\ aver la collaboration technicue de P. DACHECX \\ Station de Recherches sur l'Elevage des Ruminants, \\ Centre de Recherches de Clermont-Ferrand, I. N. R. A., \\ Theix. Saint Genès Champanelle, 63110 Beaumont
}

\section{RÉSUMÉ}

Cette expérience avait pour but la comparaison de l'avoine décortiquée, du mais et de l'orge dans les aliments concentrés de démarrage pour agneaux allaités artificiellement et précocement sevrés $\left(42^{\mathrm{e}}\right.$ jour $)$.

Trois aliments concentrés broyés et agglomérés, contenant chacun une seule des trois céréales, ont été fabriqués en granulés de $5 \mathrm{~mm}$ et de $2,5 \mathrm{~mm}$ de diamètre. Chaque aliment était distribué sous ses deux formes (granulés de $5 \mathrm{~mm}$ et de $2,5 \mathrm{~mm}$ ), en libre choix et à volonté à 5 lots de 5 agneaux entre les âges de 15 et 52 jours. Un foin de graminées distribué à volonté jusqu'à 5 semaines puis limité ensuite à $20 \mathrm{p}$. 100 de la ration était également mis à la disposition des animaux.

Les aliments "avoine décortiquéc " et "maïs " ont été ingérés en quantités équivalentes, toujours plus élevées que l'aliment "orge ", la différence entre l'orge et le mais atteignant la signification au cours de la $6^{e}$ semaine $(P<0,03)$.

L'acceptabilité des deux types de granulés a été identique avec les aliments "orge " et "avoine décortiquée "tandis que les granulés de $2,5 \mathrm{~mm}$ de diamètre étaient préférés clans le cas de l'aliment " mäis " (différence significative : $\mathrm{P} \cdots 0,05$ ).

\section{INTRODUCTION}

L'allaitement artificiel et les méthodes de conduite intensive du troupeau de brebis accroissent l'intérèt du sevrage précoce dont la réussite est conditionnée, en premier lieu, par les quantités d'aliments solides ingérées par le jeune animal. Or, le comportement de l'agneau âgé de 6 à 8 semaines vis-à-vis des aliments concentrés est mal connu et l'étude de ses facteurs de variation a rarement été abordée. Ies mesures d'appétibilité relative effectuées par différents auteurs (Rosss, 
Karr et Pavey, 1961; Light, 1965; Davies, Lerman et Crosse, 1974) permettent de proposer une classification par ordre de préférence de quelques aliments simples ou composés. Dans la pratique cependant, à l'inverse des expériences d'appétibilité, on distribue généralement un seul aliment, ce qui supprime pour l'animal la possibilité de choisir. L'aliment le plus intéressant est alors cəlui qui présente l'ingestibilité lá plus élevée c'est-à-dire celui qui, offert seul, est ingéré en quantité la plus importante. Ce n'est d'ailleurs pas toujours, comme l'a montré Lighr (I965), celui qui est le plus appétible.

Les céréales constituant la majeure partie des aliments concentrés, nous avons mesuré l'influence de trois d'entre clles, l'avoine décortiquée, ie mais et l'orge, sur l'ingestibilité des aliments de démarrage pour agneaux allaités artificiellement et précocement sevrés. Trois aliments concentrés broyés et agglomérés contenant chacun une seule des 3 céréales ont été comparés. Chaque aliment a été distribué à un groupe d'agneaux entre 15 et 52 jours d'àge et sous 2 formes différentes (granulés de 2,5 et de $5 \mathrm{~mm}$ de diamètre) pour étudier l'influence de la taille des granulés sur les quantités ingćrées.

\section{MATÉRIEL ETT MÉTHODES}

Les 3 aliments concentrés (aliment avoine décortiquée, aliment maïs et aliment orge: tableau I) ont été comparés à l'aide de 3 groupes de 25 agneaux (un groupe par aliment). ('haque groupe était constitué de 5 lots de 5 animaux qui recevaient chacun un seul des 3 aliments sous les deux formes de présentation laissées en libre choix.

Les 75 agneaux, tous croisés Berrichon $\times$ (Romanov $\times$ L.imousin), ont été séparés de leur mère entre 6 et $\mathbf{2} 2$ heures après la naissance et allaités artificiellement avec un aliment d'allaitement contenant 25 p. Ioo de matières grasses et 25 p. Ioo de matières azotées (MoLENAT et T’herifz, I972). A l'âge d'une semaine, ils ont été répartis dans leurs différents lots.

\section{TABLEAU I}

Constituants et composition des aliments concentrés utilisés Coinposition of the pelleted concontrates

Constituants (p. Ion de l'aliment) (Constituents p. 100 of the pellets)

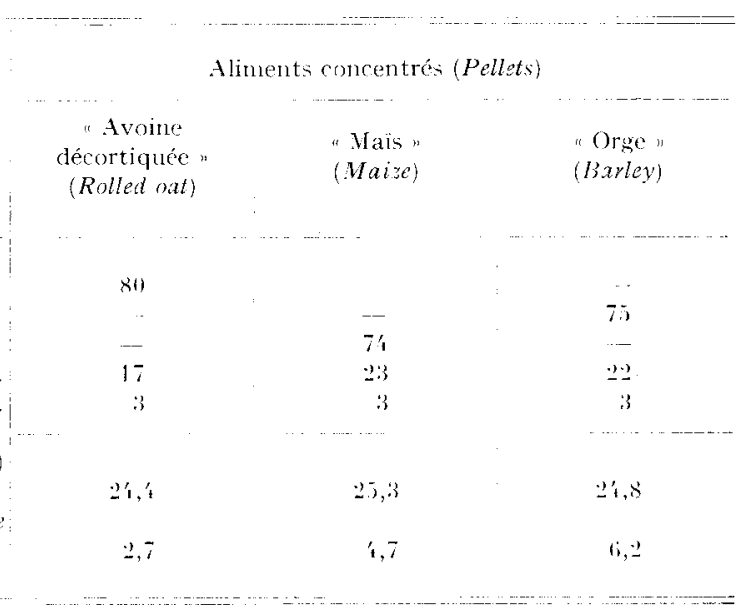

Avoine décortiquée (Rolled oat) .........

Orge (Barley) ....................

Mais (Maize) ..................

Tourtean d'arachide (Groundnut cake) ....

Complément minéral (Minerals) ........

Matières azotées totales (p. 100 de lis MS)

(Crute protein : p. $100 \mathrm{DM}$ ) ......... Cellulose brute (p. 100) de la MS) (Crude: fibre: $p, 100 \mathrm{DM}) \ldots \ldots \ldots \ldots \ldots$
Le lait de remplacement, dont la teneur en matière sèche était d'environ I6 p). IOo (MoLExAT et Theriez, 1974), était distribué à volonté jusqu'à l'àge de $2 \mathrm{I}$ jours, puis limité à $\mathrm{I}, 5 \mathrm{~kg}$ par agneau et par jour (235 g de MS) jusqu'à 35 jours. Du $36^{c}$ au $+2^{\mathrm{e}}$ jour, les quantités offertes ont été diminuées progressivement jusqu'à suppression totale. 
Dès l'àge de I 5 jours, les agneaux ont reçu à volonté, et sous les deux formes de présentation (granulés de 2,5 et $5 \mathrm{~mm}$ de cliamètre) l'aliment concentré qui leur était attribué (refus supérieurs à I 5 p. 100 des quantités offertes). I.es aliments étaient distribués chaque jour et les quantités refusées pesées trois fois par semaine. Les agneaux disposaient également d'un foin de uraminées offert à discrétion jusqu'à l'âge de 5 semaines, puis en quantité limitée, égale à 25 p. 100 de la quantité d'aliment concentré ingérée au cours des 3 jours précédents. Lés agneaux étaient logés sur litière de paille et disposaient al'eau en permanence.

Pour éviter un éventuel phénomène d'accoutumance, la disposition des cleux types de granulés était changée à chaque pesée de refus, soit 3 lois par semaine.

L'expérience s'est poursuivie jusqu'à l'âge de 52 jours, c'est-à-dire jusqu'à ce (jue les quantités moyennes d'aliment concentré ingérées aicnt atteint, dans tous les régimes, au moins joo g par animal et par jour.

Les agneaux ont été pesés à la naissance, puis chaque semaine au cours ale l'expérience et aussi à la fin de l'expérience (53 jours).

Pour la comparaison des régines, on a utilisé une analyse de variance qui a porté sur les individus dans le cas des gains de poicls vif et sur les lots dé 5 agneaux dans le cas des quantités ingérées.

\section{RÉSLITATS E'T DISCUSSION}

()n peut distinguer cleux périodes dans cet essai : la première avant 35 jours d'age moyen des animaux) correspond à la phase lactée : la deuxième (entre 35 et 52 jours) représente là phase de sevrage et de post-serrage.

Pendant la phase lactée, les auneaux ont ingéré de très faibles quantités d'aliment; solides (tabl. 2, fig. I). Il a fallu attendre le $3 t^{20}$ jour pour que la quantité quotidienne de matière sèche d'aliment concentré ápasse, dans tous les lots, fo g par agneau. An cours de cette période, les différences entre les 3 aliments ont été peu marquées (tabl. 2); seul l'aliment "avoinc décortiquée" semble avoir été légèrement mieux apprécié, principalement avant 30 jours, peut-être par suite d'unc dureté moindre. Ie foin a été consommé en quantité presque aussi importante que l'aliment concentré. Ia proportion de foin volontairement ingérée serait donc plus élevée clans la ration des agncaux très jeunes que dans celle des agneaux âgés de plus de g somaines pour leaquels on obsirve des rapports foim/aliment concentré de l'ordre de zo p. Ioo (I'Not et JARRIGE, I968). (HARLetLERY, LEROY et ZELTER (I954) avaient déjà ofservé wn phénomène analogue sur des agneaux sevés très précocement.

Entre 35 et 52 jours, la quantité moyenne d'aliment concentré ingérée a augmenté rapidement pour les 3 régimes mais clle est demeurée constamment plus latble arec l'aliment "orge "qu'avec les deux autres (iig. I). Les différences par rapport aux aliments "avoine lécortiquée " ot "maïs", qui sont respectivement de 9,5 et 13 p. 100 , ne sont pas significatives (tabl. 2). lilles sont plus importantes pendant la première semaine de cutte période (Ge scmaine l'âge des agneaux) au cours de laquelle l'aliment liquide a été progressivement supprimé : respectivement $I, t^{0} \pm 0,2 \mathrm{I}$; $\mathrm{I}, 24=0,08$ et $\mathbf{I}, 09=0,32 \mathrm{~kg}$ de matière sèche par aşneau et pour la semaine avec les aliments "maïs ". "avoine décortiquée net "orgen (différence significative entre " orge " et " maïs ": $P$. 0,03 ).

L'effet de la taille des granulés s'est manifesté différemment sclon les régimes. Alors qu'avec les aliments "avoine décortiquée "et "orge "les agneaux ont été inclifférents à la forme de présentation, ils ont préféré les granulés de $2,5 \mathrm{~mm}$ à ceux de $5 \mathrm{~mm}$ dans le cas de l'aliment " maïs" (P 0,05, tabl. 2) peut-ètre parce que ces derniers étaient plus durs (dureté mesurée par compression dynamométrique). De la même façon. Aumartre et SALMON-Lligagneur (igor) ont observé, avec un aliment à base de blé, que les porcelets préléraient les granulés de $2,5 \mathrm{~mm}$. Pourtant avec l'aliment "orge " cette différence n'est pas apparue bien que les granulés fussent aussi durs que pour l'aliment " maîs ". Il est possible que la présence d'une quantité plus importante d'enveloppes de grains clans l'aliment "orge ncrée des zonés de discontinaité plus fréquentes qui rendent les granulés plus friables dans la bouche. 


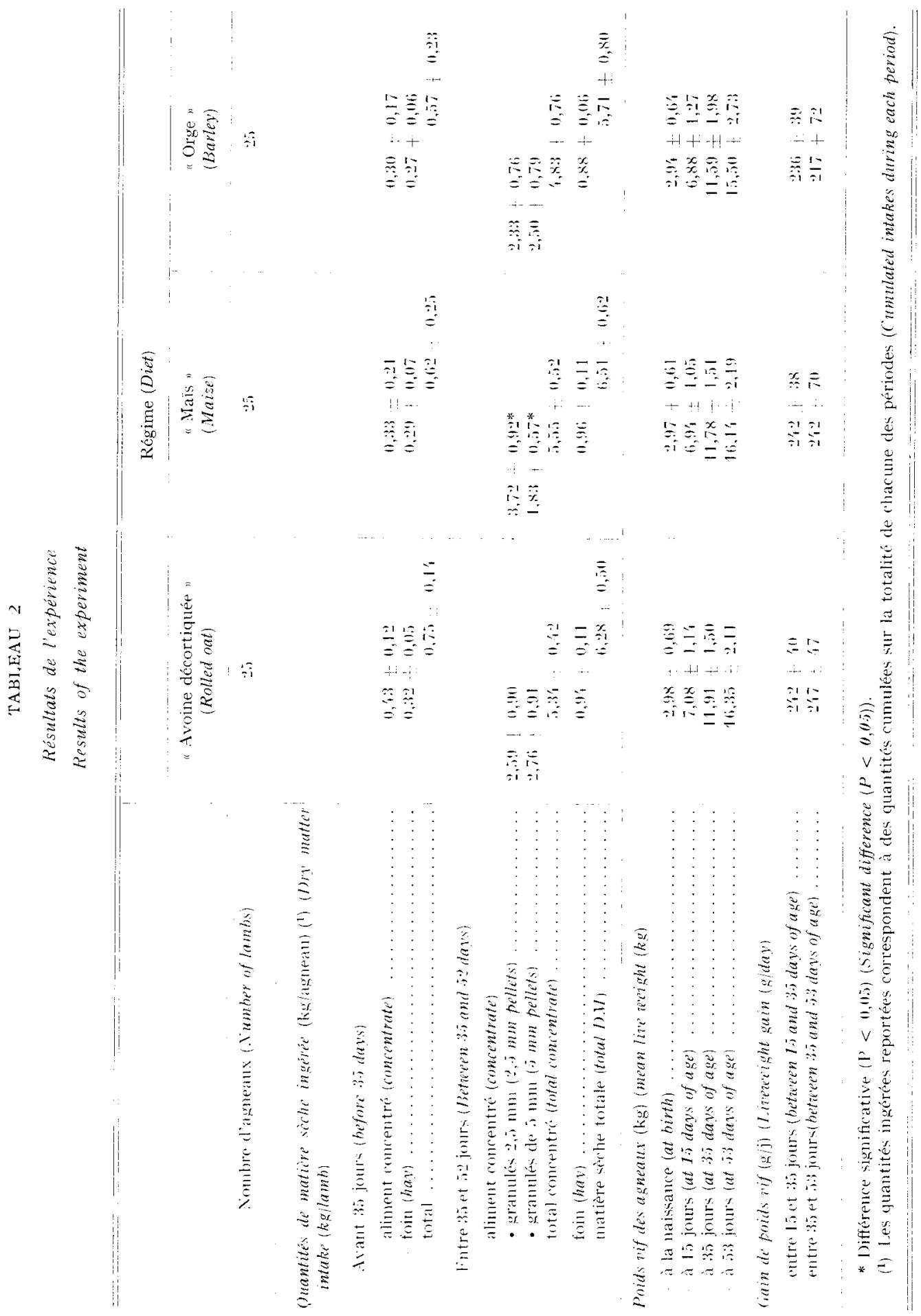


lendant toute la durée de l'essai, les aliments " maïs "et "avoine décortiquée " ont été ingérés en quantités équivalentes et plus élevées que l'aliment "orge ". Le clasiement ainsi obtenu est contraire à celui observé dans les expériences de mesure d'appétibilité des différentes céréales offertes simultanément, soit entières, soit concassées, expériences dans lesquelles le maïs et l'avoine apparaissaient moins appréciés que l'orge (Light, 1965: Davies, Liknax et Crosse, 1974). Dans ces dernières expériences, la forme de présentation a pu jouer un rôle important, le maïs concassé ou en flocons utilisé étant de préhension difficile et sa dureté rebutant les agneaux. Il semble bien que la forme de présentation est particulièrement importante dans le cas du maïs.

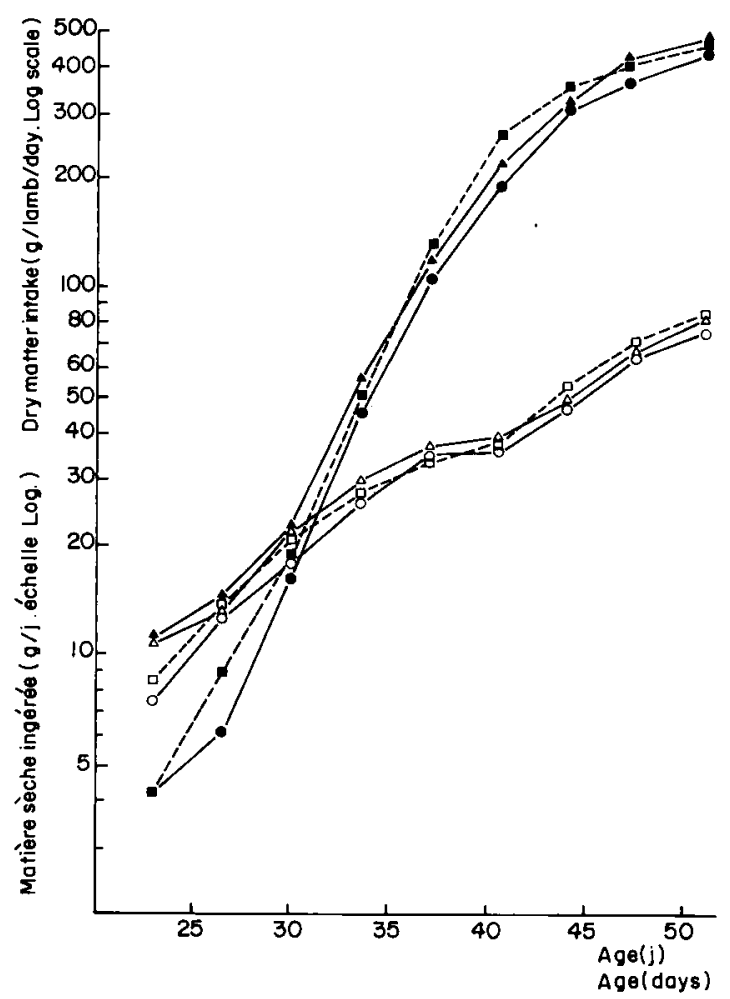

FIx. I. Frolution des quantites de matiere sche ingérés (Moyennes journalières par agneau)

Mean daily intakes of hay and concentrate dry matter

\begin{tabular}{|c|c|c|}
\hline $\begin{array}{l}\text { Ints avoine décortiquée } \\
\text { (rolled oat groups) }\end{array}$ & $\left\{\begin{array}{l}\text { foin } \\
\text { concentre }\end{array}\right.$ & $\begin{array}{l}\triangle \longrightarrow \triangle \text { hay } \\
\Delta \longrightarrow \text { concentrate }\end{array}$ \\
\hline $\begin{array}{l}\text { lots maiss } \\
\text { (maize groups) }\end{array}$ & $\left\{\begin{array}{l}\text { foin } \\
\text { concentre }\end{array}\right.$ & $\begin{array}{l}\square-\ldots+\cdots \text { hay } \\
-\ldots-\ldots \text { concentrate }\end{array}$ \\
\hline $\begin{array}{c}\text { lots orge } \\
\text { (barley groups) }\end{array}$ & $\left\{\begin{array}{l}\text { foin } \\
\text { roncentro }\end{array}\right.$ & $\begin{array}{l}\text { - hay } \\
-\square \text { concentrate }\end{array}$ \\
\hline
\end{tabular}

L'influence de la taille des granulés observée dans la présente expérience, est à rapprocher en effet des résultats de Dickson et Walker-Love (1967) qui ont rapporté que le maïs moulu grossièrement (semoule) était mieux apprécié, par des agneaux plus âgés il est vrai, que l'orge ou le maïs entiers.

Mais lorsqu'on supprime à l'animal la possibilité de choisir, les facteurs d'appétibilité diminuent en importance et la régulation de la quantité ingérée s'effectuc vraisemblablement en 
priorité par l'intermédiaire de phénomènes d'ordre digestif et à un deyré moindre, surtıut en période de sevrage, par des phénomènes d'ordre métabolique. Ainsi, offert seul pendant le sevrage, le mais, qu'il soit concassé (Light, 1965) ou broyé-aggloméré, est ingéré en quantité plus élevée que l'orge. Le mais étant en outre plus riche en énergie, se révèle donc très avantageux au sevrage car il permet de diminuer le déficit éncrgétique des jeunes lorsque le lait est supprimé.

Tout au long de l'expérience, les gains de poids vil ont reflété assez fidèlement les différences observécs sur les quantités ingérées. Ils ont été peu différents d'un groupe à l'autre avant 35 jours (tabl. 2). Au-delà, la croissance des agneaux recevant l'aliment "orge " a été très in lérieure à celle des autres, particulièrement au cours de la $6^{\circ}$ semaine $(149+75$ contre 181 t. 70 et 182 土 107 $\mathrm{g} / \mathrm{j}$ pour l'avoine et le mais) mais la variabilité individuelle est très grande et aucune des différences n'est significative.

En définitive, le maïs se révèle supérieur à l'orge dans les aliments de démarrage pour agneaux, aussi bien en ce qui concerne l'ingestibilité que la croissance. De même, l'avoine décortiquéc, sensiblement à égalité av'ec le maîs, est supérieure à l'orge, mais le décorticage accroît son coût dans de telles proportions qu'elle n'est plus guère employée, même dans l'espèce porcine. Le mais peut donc être considéré actuellement comme la céréale la plus intéressante pour l'alimentation de l'agneau puisqu'elle est également la mieux consomméc pendant l'engraissement (HANKF et Jordan, I963; Agefr, Therifz et Molexat, ig7il.

Recu pour publication on juillet 195.5.

SUMMARY

COMPARISON OF THE VOLUNTARY INTAKE OF DEHUI,IED OAT, BARLEY AND MAIZE IN CONCENTRATE FEEDS FOR EARI Y WEANED IAAMBS

The aim of the present trial was to compare dehulled oat, barley and maize in the starter concentrates for artificially reared and early weaned lambs ( 2 th day).

A total of 75 lambs were used in this study. They were divided into 15 groups of 5 animals each and received a milk replacer ad libitum up to 3 weeks and then in restricterl amounts ( $\mathrm{I} .5 \mathrm{~kg} / \mathrm{lamb} /$ day) till the age of 5 weeks. During the $6 \mathrm{th}$ week. the amount of milk replacer was progressively reduced in order to wean the lambs at 6 weeks.

Three concentrates, each containing only one of the three cereals (table 1 ), were processed into pellets with a diameter of $5 \mathrm{~mm}$ and $2.5 \mathrm{~mm}$. Each concentrate was offered in these two forms ( $5 \mathrm{~mm}$ and $2.5 \mathrm{~mm}$ pellets) in free choice and ad libitum to 5 groups of 5 lambs from the age of two weeks. Grass hay was also available for the animals, ad libitum before 5 wecks and then restricted to $20 \mathrm{p}$. 100 of the diet. The trial was finished to days after suppression of the milk replacer, when the lambs werc, on an average, 52 days old.

Before 3.5 days, the amounts of solid lecd eaten by the lambs were small and the differences between the 3 diets were only slightly marked (table 2 ). The intake of the "lehuller oat diet" was a little higher than that of the others. The average weight gains were comparable for the three diets.

Between 35 and 52 days, the intakes of the "dehulled oat diet " and "maize diet "were equivalent and always higher than that of the "barley diet "(table 2), but none of the differences were significant. During the weaning week (lambs aged 6 weeks), the differences were larger : I. $09+0.32 ; \mathrm{I} .24=0.08 ; \mathrm{I} .46=0.2 \mathrm{I} \mathrm{kg}$ dry matter respectively per lamb lor the "barley "dehulled oat" and "maize "diets (significant difference between " barley " and " maize ": $p<0.03$ ). Consequently, the animals which received the "barley diet " exhibited the poorest weight gains.

The intakes of the two types of pellets were identical for "barley " and "dehulled oat " diets, whereas $2.5 \mathrm{~mm}$ pellets were preferred in the case of the " maizc dict "(significant difference: $p<0.05)$.

It may be concluded that, at the present time, maize represents the most favourable cereal for lamb rearing because of its high voluntary intake level both at weaning and during the fattening period. 


\section{RÉFÉRENCES BIBLIOGRAPHIQUES}

AGver D., Therliz M., Mlolenat G., ig71. Utilisation comparée du blé, de l'orge et du maís dans la ration des agneaux à l'engrais. Ann. Zootech., 20, 465-475.

Avmaitre A., Salmon-Legagnevr E., I96I. Les préférences alimentaires du porcelet. V. Comparaison de divers modes de distribution de l'aliment. Ann. Zootech., 10, r97-20.3.

Charley-l.kr Geneviève, Leroy A. M., Zelter S. Z., I954. Élevage artificiel de l'agneau précocement sevré. Essai de détermination de ses dépenses de croissance. Ann. Zootech., 3, I69-187.

J avies D. A. R., Lermax P. M., Crosse M., I974. Food preferences after weaning of artificially reared lambs. J. Agric. Sci., 82, 469-47I.

Dickson 1. A., Walker-Love J., rg67. The palatibility of certain feedingstuffs and mixtures for blakface lambs. Anim. Prod.,, $282 \mathrm{~A}$.

MANKE H. E. et Jordan R. M., I963. Comparison of lambs fed shelled corn and whole or pelleted barley of different bushel weights. J. Anim. Sci, 22, ro97-1099.

LiGHT M. R., I965. What baby lambs prefer to eat. North Dakota Farm Research., 23 (II), 23-24.

Molenat G., Theriez M., 1972. L'allaitement artificiel des agneaux. II. Influence de la teneur en matieres grasses du lait de remplacenent. Ann. Zootech., 21, 385-399.

Molenat G., Theriez M., I974. L'allaitement artificiel des agneaux. $V$. Comparaison de différentes concentrations du lait de remplacement. Ann. Zootech., 23, 491-502.

PINOT R., JARRIGE R., r968. Utilisation des aliments broyés et agglomérés par l'agneau à l'engrais. II. Comparaison du foin de luzerne condensé au foin de luzerne normal en présence d'un aliment concentré offert ad libitum. Ann. Zootech., 17, 5-22.

Ross C. V., Karr M. L., PAvir R. L., I96r. Creep feeding studies with lambs. Missonti t gricultural experiment station. Research Bulletin., rya, 2-16. 